\title{
DAVINCI: Deep Atmosphere Venus Investigation of Noble gases, Chemistry, and Imaging
}

\author{
Lori S. Glaze, James B. Garvin, Brent Robertson, Natasha M. Johnson, Michael J. \\ Amato, Jessica Thompson, Colby Goodloe, Dave Everett and the DAVINCI Team \\ NASA Goddard Space Flight Center, Code 690 \\ 8800 Greenbelt Road \\ Greenbelt, MD 20771 \\ 301-614-6466 \\ Lori.S.Glaze@nasa.gov
}

\begin{abstract}
DAVINCI is one of five Discovery-class missions selected by NASA in October 2015 for Phase A studies. Launching in November 2021 and arriving at Venus in June of 2023, DAVINCI would be the first U.S. entry probe to target Venus' atmosphere in 45 years. DAVINCI is designed to study the chemical and isotopic composition of a complete crosssection of Venus' atmosphere at a level of detail that has not been possible on earlier missions and to image the surface at optical wavelengths and process-relevant scales.
\end{abstract}

\section{Table of Contents}

1. INTRODUCTION 1

2. MISSION DESIGN................................................... 2

3. PAYLOAD ...................................................... 2

4. SUMMARY ...................................................... 3

ACKNOWLEDGEMENTS .......................................... 3

REFERENCES..................................................... 3

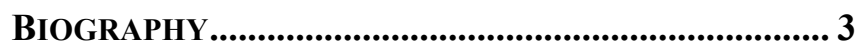

\section{INTRODUCTION}

Venus and Earth experienced vastly different evolutionary pathways resulting in unexplained differences in atmospheric composition and dynamics, as well as in geophysical processes of their planetary surfaces and interiors. Understanding when and why the evolutionary pathways of Venus and Earth diverged is key to understanding how terrestrial planets form and how their atmospheres and surfaces evolve. DAVINCI (Figure 1) would provide these missing puzzle pieces needed to understand terrestrial planet formation and evolution within the solar system and beyond. The DAVINCI mission is tightly focused on answering fundamental questions that have been ranked as high priority by the last two National Research Council (NRC) Planetary Decadal Surveys [1-3] as well as by the Venus Exploration Analysis Group (VEXAG) since the time of its inception in 2005 [4]. For example, DAVINCI will make measurements of the heaviest noble gases, including the first ever measurements of xenon [5] and its isotopes. These definitive measurements, which would be made well below the homopause to avoid any ambiguity, are sufficient to answer

U.S. Government work not protected by U.S. copyrigh questions as framed by the NRC Planetary Decadal Survey and VEXAG, without the need to repeat them in future New Frontiers or other Venus missions.

The three major DAVINCI science objectives are:

- Atmospheric origin and evolution: Understand the origin of the Venus atmosphere, how it has evolved, and how and why it is different from the atmospheres of Earth and Mars.

- Atmospheric composition and surface interaction: Understand the history of water on Venus and the chemical processes at work in the lower atmosphere.

- Surface properties: Provide insights into tectonic, volcanic, and weathering history of a typical tessera (highlands) terrain.

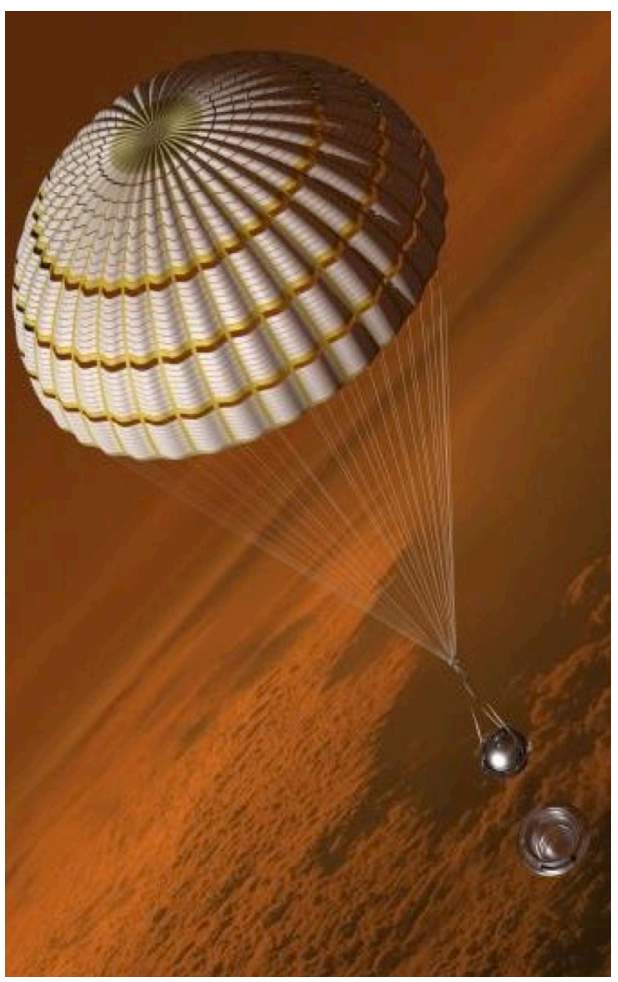

Figure 1. DAVINCI will probe the Venus atmosphere. 


\section{MISSION DESIGN}

The DAVINCI probe will make in situ measurements during its one-hour descent through the Venus atmosphere. The descent sphere itself builds on the successful Pioneer Venus Large Probe design, flown in 1978. The descent sphere and payload are protected during initial atmospheric entry by a solid body aeroshell.

The DAVINCI probe would be delivered to Venus by a carrier/telecommunications spacecraft built by Lockheed Martin. The spacecraft first encounters Venus four months after launch. This initial flyby enables targeting, when the spacecraft returns to Venus 15 months later, of the probe atmospheric entry location for optimized lighting conditions over the tessera region chosen for descent imaging observations. The probe is released a few days before the second Venus encounter and the spacecraft communicates directly with the probe throughout coast, entry, and descent. The probe employs a two-parachute system to extract the descent sphere from the entry system and to slow descent.

The entire science payload is contained within the pressure and temperature controlled descent sphere. All science data are collected and relayed to the flyby spacecraft during descent (Figure 2). DAVINCI has no requirement to survive touchdown; however, the descent sphere carries sufficient resources (e.g., power, thermal control) to continue science operations and data relay for $\sim 17$ minutes on the surface. After loss of contact with the probe, the spacecraft relays all data back to Earth multiple times.

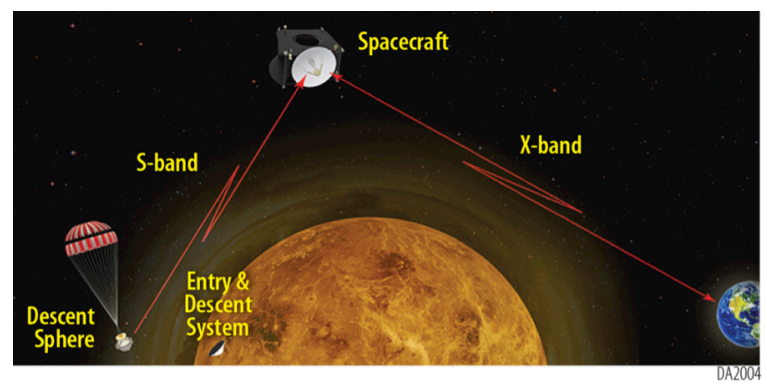

Figure 2. The Spacecraft acts as a communication relay between the Descent Sphere and Earth.

\section{PAyload}

DAVINCI builds on the tremendous success of the Mars Science Laboratory Sample Analysis at Mars (MSL/SAM) suite carried on the Curiosity rover [6-13], by pairing the Venus Mass Spectrometer (VMS) led by Melissa Trainer and Paul Mahaffy at NASA's Goddard Space Flight Center with the Venus Tunable Laser Spectrometer (VTLS) led by Chris Webster and Jordana Blacksberg at the Jet Propulsion Laboratory. These two instruments (Figures 3 and 4) provide the first comprehensive measurements of noble and trace gas species, as well as key elemental isotopes.

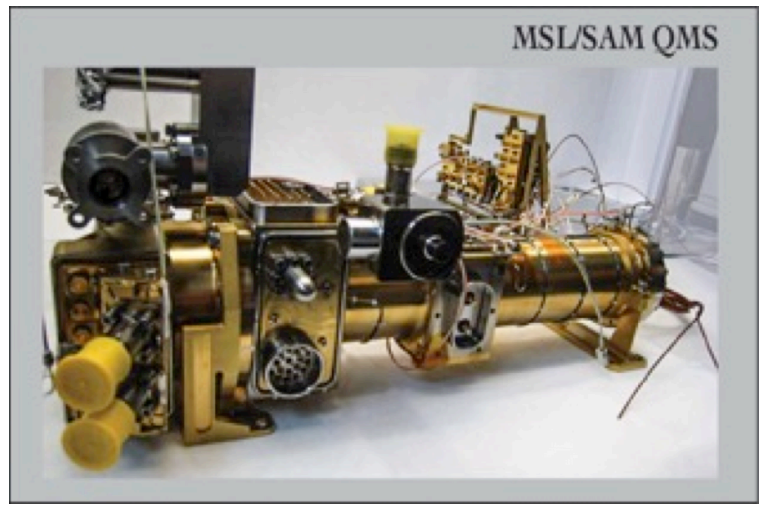

Figure 3. The VMS has a rich heritage that includes the only noble gas measurement at Jupiter during the Galileo Probe descent and the first precision measurements of argon, krypton, and xenon isotopes on Mars with the MSL/SAM Quadrapole Mass Spectrometer.

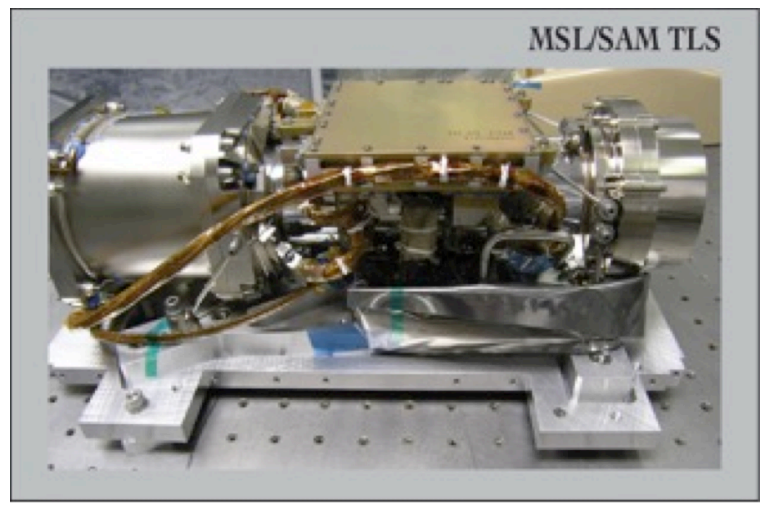

Figure 4. The VTLS builds on the success of the MSL/SAM Tunable Laser Spectrometer, using flight proven laser and optics that enable precision measurements of key trace gases.

These two state-of-the art instruments are complemented by the Venus Atmospheric Structure Investigation (VASI). VASI (Figure 5) provides measurements of the structure and dynamics of the Venus atmosphere during entry and descent, including the first detailed profile of the deep atmosphere. These observations will be used as context for the chemistry measurements, and enable reconstruction of the descent profile. Science leadership for VASI is provided Ralph Lorenz at the Applied Physics Laboratory and Dave Atkinson at the Jet Propulsion Laboratory. The sensor suite is provided by NASA Goddard Space Flight Center.

The Venus Descent Imager (VenDI) provides high-contrast descent imaging of the tessera terrain (Figure 6) at scales not possible from orbit. Led by Mike Ravine of Malin Space Science Systems, it leverages successful flight experience from the MSL Curiosity Rover's Mastcam and MARDI descent video imaging systems. Its planned imaging 
sequence will bridge the resolution-gap from orbital radar images (Magellan) and landed panoramas (Soviet Venera landers), while also enabling local scale topographic measurements.

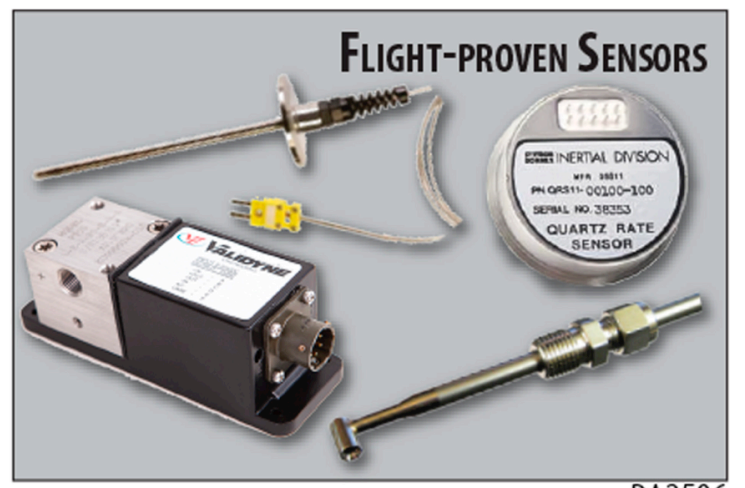

Figure 5. VASI employs flight-proven commercial sensors to measure atmospheric temperature, pressure, acceleration and angular motions. VASI also uses the S-band communication link to measure atmospheric winds via Doppler tracking.

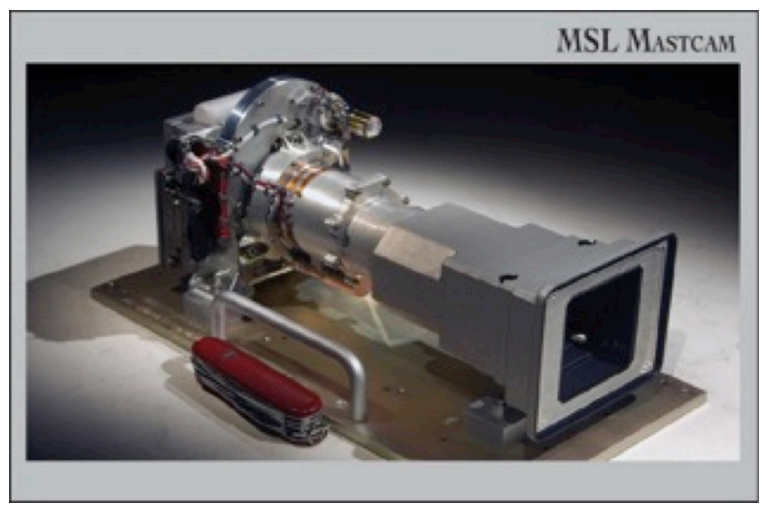

Figure 6. VenDI leverages a distinguished record of high quality, flight-proven planetary imagers, with recent heritage from MSL's Mastcam and MARDI.

\section{SUMMARY}

The DAVINCI atmospheric probe will leverage flightproven 21 st Century instrument technology to definitively resolve key Venus atmospheric science questions. Imaging of tessera at scales relevant to future landers $(1-10 \mathrm{~m})$ will resolve radar ambiguities and uncertainties, while also providing boundary conditions for geologic models of the highlands. DAVINCI will meet multiple, high-priority National Academy of Sciences goals, while also serving as a pathfinder for future orbital radar missions and landed surface composition missions to the Venus highlands.

\section{ACKNOWLEDGEMENTS}

The authors thank the NASA's Planetary Science Division for Phase A funding for this project. Special thanks to Noel Hinners for inspiring the 9 years of effort that has led to a mature Venus atmospheric probe mission (DAVINCI).

\section{REFERENCES}

[1] Crisp, D., et al. (2002) ASP conference Series, 272, Ed. MV Sykes, 5-34.

[2] New Frontiers in the Solar System (2003) National Research Council of the National Academies, National Academies Press.

[3] Visions and Voyages (2011) National Research Council of the National Academies, National Academies Press.

[4] VEXAG (2014) Goals, Objectives, and Investigations for Venus Exploration, http://www.lpi.usra.edu/vexag/reports/GOI-140625.pdf.

[5] Trainer et al. (2016) International Venus Conference, Oxford.

[6] Mahaffy et al. (2015) Science, 347, 412-414.

[7] Webster et al. (2015) Science, 347, 415-417.

[8] Atreya et al. (2013) GRL, 40, 5605-5609.

[9] Mahaffy et al. (2013) Science, 341, 263-266.

[10] Webster et al. (2013) Science, 341, 260-263.

[11] Wong et al. (2013) GRL, 40, 6033-6037.

[12] Conrad et al. (2016) EPSL 454, 1-9.

[13] Webster et al. (2016) International Venus Conference, Oxford.

\section{BIOGRAPHY}

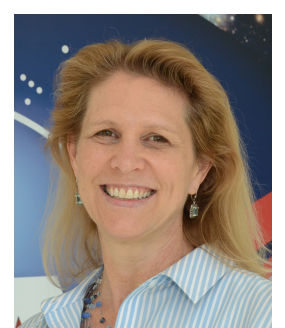

Lori S. Glaze is the Deputy Director for the Solar System Exploration Division at NASA's Goddard Space Flight Center. She received Bachelors (1985) and Masters (1988) degrees in Physics from the University of Texas (Arlington), and a PhD (1994) in Environmental Science from Lancaster University (England). Prior to coming to Goddard, Dr. Glaze was Vice President of Proxemy Research, a private research firm, and also worked at the Jet Propulsion Laboratory. Her research has focused on developing unique theoretical models for the dynamics of explosive volcanic eruption plumes and the emplacement of lava flows on the 
Earth, Venus, Mars, and Io. Over the last ten years, she has become increasingly involved in multiple efforts to return NASA to Venus. Dr. Glaze has served as the Chair of the Venus Exploration Analysis Group (VEXAG) and as a member of the NASA Advisory Council's Planetary Science Subcommittee (2013 - 2016). Dr. Glaze is currently the Principal Investigator of the DAVINCI mission, which is one of five concepts undergoing Phase A study in NASA's Discovery Program.

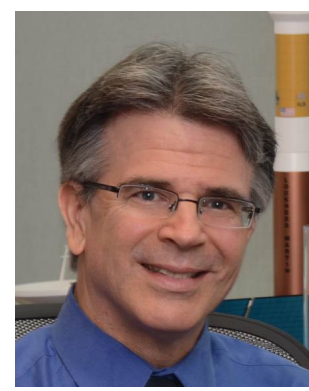

James B. Garvin is the Chief Scientist of NASA's Goddard Space Flight Center. He received his Bachelor's degree from Brown University (1978) and a Masters from Stanford (1979), and then a Ph.D. from Brown University (1984), with emphasis on the geological evolution of the surfaces of Venus and Mars. Prior to his current position, he served NASA as Agency Chief Scientist, Chief Scientist of Mars Exploration, and as Project Scientist for the Earth System Science Pathfinder Program. He is presently a Co-investigator on the Mars Curiosity rover imaging team, with flight experience associated with the MARDI descent imaging system. In addition, he chaired the NASA Administrator's Decadal Planning Team to develop strategies for integrated human and robotic exploration of the Solar System. In 2004, he appeared on the Late Show with David Letterman. Dr. Garvin is the Deputy Principal Investigator for DAVINCI and serves Dr. Glaze as the science theme leader for the surface properties investigation associated with the Venus tessera.

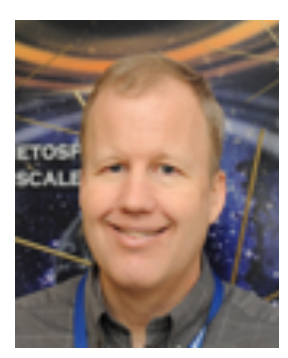

Brent Robertson is a NASA certified Project Manager at NASA's Goddard Space Flight Center. He received his Bachelor's degree (1984) in Engineering Science from University of Toronto and Master's degree (1985) in Aerospace Engineering from the University of Virginia. Mr. Robertson has over 30 years of aerospace industry experience, including 13 years in program and project management. Most recently, he was detailed to NOAA as the Joint Polar Satellite System (JPSS) Program Implementation Manager. Mr. Robertson was the Deputy Project Manager for Magnetospheric Multiscale (MMS) that consisted of four spacecraft built in-house at GSFC with a contracted suite of 100 instruments. He also served as Observatory Manager for GSFC's in-house developed Solar Dynamics Observatory. Mr. Robertson is currently the DAVINCI Project Manager.

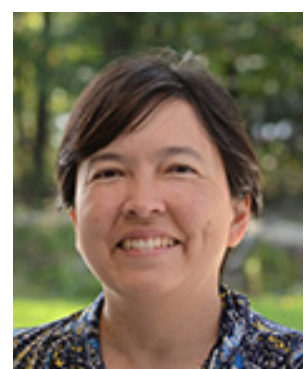

Natasha M. Johnson is a Research Space Scientist in the Astrochemistry Laboratory at NASA Goddard Space Flight Center. She received her Bachelors from the University of Arizona (1991) and both her Masters (1998) and PhD (2002) from Washington University in St. Louis. Her graduate research emphasized hydrous mineral stability on the surface of Venus. Prior to landing at Goddard as a postdoc in 2002, Dr. Johnson lived and worked at Mount Wilson Observatory in California as lead solar observer of the 60ft Solar Tower, was a museum guide at Griffith Observatory in Los Angeles, and worked briefly with both Voyager and Ulysses missions. Her current research involves experimental work researching the early solar nebula and the surface of Venus. Dr. Johnson is the Project Scientist for DAVINCI and will support the PI and mission as a liaison between the science and engineering teams.

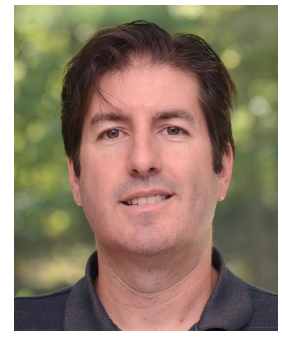

Michael J. Amato is a systems engineer at NASA's Goddard Space Flight Center. He is the manager and lead engineer for Goddard's Planetary line of business. He has a bachelor's degree in aerospace engineering and a graduate degree in systems engineering. Michael has worked probe missions, entry probe designs and prototype testing for a large part of the past decade. Michael has over 25 years of experience developing and building a variety of flight instruments and missions at NASA, working in both the science and engineering directorates at Goddard.

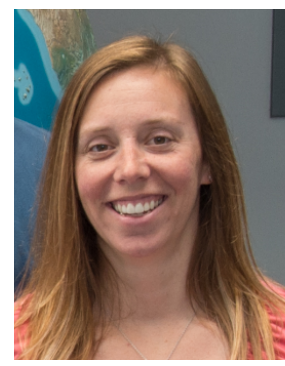

Jessica Thompson is currently the Deputy Project Systems Engineer of the DAVINCI mission, which is one of five concepts undergoing Phase A study in NASA's Discovery Program. She received a Bachelor's degree (2001) in Physics from Salisbury University. Ms. Thompson has served as a systems engineer for multiple missions including Plankton, Aerosol, Cloud, ocean Ecosystem (PACE), LandSat 8 Thermal InfraRed Sensor (TIRS), and the recently successful Magnetospheric MultiScale (MMS) mission, where she focused on fault management and RF communications. Her career at NASA began in 2002 in NASA Wallops Flight Facility's Guidance, Navigation and Control and Mission Systems Engineering Branch. She served as an electronics engineer for multiple suborbital payloads including the Max Launch Abort System (MLAS), the Autonomous Flight Safety System (AFSS), and led student teams to develop and launch payloads on multiple platforms including CubeSats and scientific balloons 


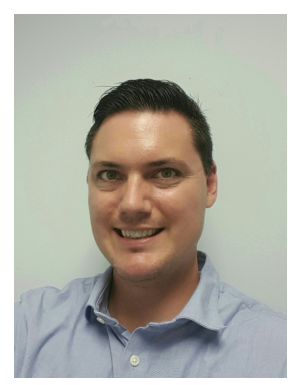

Colby Goodloe received a B.S. and M.S. in Electrical Engineering from University of Maryland in 2005 and 2008, respectively. He has worked for NASA Goddard Space Flight Center since 2002, while working as a coop student on GNC components. After graduating, he continued to work on the electronics for GNC components and instruments for several successful missions including SDO, GPM, FASTSAT, MMS, and 7-SEAS BASELInE and others still in development including ICESAT-2, and $L C R D$. In the past several years he has transitioned to the systems engineering discipline as a Project $V \& V$ engineer for OSIRIS-REx and the Venus Operations Phase Lead for DAVINCI. He enjoys spending time with his wife and two kids, outdoor activities, and sports.

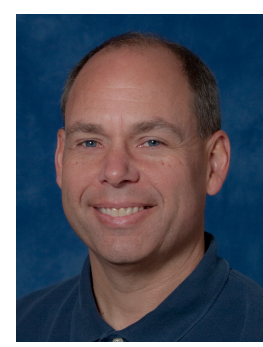

David Everett is currently the Project Systems Engineer for the OSIRIS-REx asteroid sample return mission. In his 25 years at NASA, he has led the design, build, and launch of four spacecraft, and he was a key player during the launch of three others. Before OSIRIS-REx, Mr. Everett led the technical effort for the Lunar Reconnaissance Orbiter as the Mission Systems Engineer, from design through early-orbit operations. Mr. Everett has actively supported NASA outreach activities through over 75 speaking engagements. He has received 37 individual awards, 25 group awards, and a patent for his efforts at NASA; he has published 18 papers; and he coedited (and wrote the spacecraft design chapter for) the book Space Mission Engineering: The New SMAD. He earned a BSEE summa cum laude, at Virginia Tech in 1986 and a MSEE at the University of Maryland in 1989. Before he joined NASA in 1991, Mr. Everett worked at Westinghouse Electric Corporation where he was awarded two patents for his designs of RF circuits. 